\title{
Fuzzy model for human autonomous computing in extreme surveillance and it's applications
}

\author{
Varatharajan Ramachandran* \\ Bharath University, India
}

\begin{abstract}
This special issue of the Journal of Intelligent \& Fuzzy Systems contains selected articles of Fuzzy model for human autonomous computing in extreme surveillance and it's applications
\end{abstract}

\section{Introduction}

Article [1] helps to improve the intuitiveness of the stock forecasting model, based on the image recognition technology; this study normalizes the image and performs feature recognition with grayscale images. At the same time, this paper ignores the small fluctuations and combines the characteristics of stock images to remove the drying process and proposes an algorithm model based on feature recognition. In Article [2] a smart drinking water management strategy for water supply based on sensors, comparator circuits, PLC (Programmable logic control) and fuzzy logic is suggested to control the water supply in order to reduce water usage and achieve an equative sharing. Article [3] proposes Automatic number plate authentication, a mass tracking system that captures vehicles' images and authenticates their license number. Here, the Type2 Fuzzy based Tesseract Text Relative Recognition Algorithm (TTRR) obtains the image dataset from the JSON (JavaScript Object Notation) file and processes it through the link in the dataset. Article [4] presents an automated lung cancer detection system equipped with machine learning algorithm, which can differentiate between

\footnotetext{
*Corresponding author. Varatharajan Ramachandran, Bharath University, India. E-mail: drvr@bharathuniv.ac.in.
}

the benign, malignant and normal classes of lung cancer. The accuracy of the proposed lung cancer detection method is around $98.7 \%$, which is superior to the compared approaches. Article [5] proposes a system measurement of glucose level and Prediction/ diagnosis of diabetes based on the real time low complexity neural network implemented on a wearable device. Article [6] studies the Text Sentiment Analysis, and suggests a system for grading feelings based on a lexicon. Bag-of-words (BOW) or lexiconbased methodology is currently the main standard way of modeling text for machine learning in sentiment analysis approaches. Marketers can use this to analyze their business and services, public opinion, or to evaluate customer satisfaction. Article [7] studies in improving the operation efficiency of the logistics system, based on the improved neural network algorithm by combining the logistic regression algorithm to construct a logistics demand forecasting model based on the improved neural network algorithm. Article [8] constructs a monetary policy analysis model based on dynamic stochastic general equilibrium methods to analyze the interactive effects of monetary policy and other policies. Article [9] studies the structure of motor brain-computer and improves it based on traditional methods. It also uses a DSP filter to convert the preprocessed EEG signal 
$\mathrm{X}$ into a time series, and adjusts the distance between the time series to classify the data. Article [10] combines the actual situation of the financial supply chain, improves the traditional machine learning algorithm, and takes the actual company as an example to build a corresponding risk factor recognition model.

Article [11] proposes a research on inventory model based on consumer web search. This paper by considering consumers and retailers characteristics and situations an inventory model is constructed in the online search environment, and analyzes the ordering strategy. Article [12] analyzes the supplier's precision marketing status and existing problems and uses its own development and management characteristics to improve marketing strategies. Article [13] constructs a stock return influencing factor analysis model to statistically describe the sample data and factor data, and tests the applicability of the fivefactor model for performance evaluation of mixed stocks. Article [14] builds a comprehensive evaluation model of logistics enterprise competitiveness based on SEM model based on machine learning technology. Article [15] introduces the genetic algorithm, to analyze the demand for the heterogeneity of inflation expectations and explains the basic steps to use it and how to apply it to explain problems in economics. Article [16] constructs a real estate bubble financial risk analysis model based on machine learning and factor analysis models. Article [17] adopts the Bayesian estimation method based on the Minnesota prior to obtain the posterior distribution of each parameter of the VAR model. Moreover, this paper uses methods based on Kalman filtering and Kalman smoothing to obtain the posterior distribution of latent state variables. Article [18] improves traditional machine learning algorithms and builds an entrepreneurial policy analysis model based on improved machine learning to analyze the impact of entrepreneurial policies on employment. Article [19] constructs a neural network-based precision marketing model and focuses on data mining to study user churn prediction and user value enhancement, which are the two most important factors affecting marketing revenue. Article [20] builds a logistics enterprise performance evaluation system based on the nonradial and non-angle network SBM model based on machine learning algorithms.

Article [21] combines the actual needs of financial analysis to construct a financial risk spillover effect model based on ARMA-GARCH and fuzzy calculation. The model uses ARMA-GARCH and fuzzy algorithm to verify the financial multiple risk factors. Article [22] combines machine learning to construct a monetary policy differentiation effect analysis model based on the GVAR model. Article [23] combines artificial intelligence technology to analyze the impact of inflation expectations, and constructs the central bank information disclosure index and inflation expectations index. Article [24] studies the nature of the part and tail probability of dependent random variable sequences with different distributions, and focuses on the random variable sequences with wide dependent structures, and obtains the relevant probability estimation formulas. Article [25] constructs an improved neural network-based corporate bond spread model to explore the impact of macro systemic risks on credit spreads. Article [26] studies the causes of poverty with the rapid development of Internet technology and big data technology; and also, with the application of large-scale data technology and data extraction algorithms the truly poor households can be identified faster and more accurately. Article [27] reviews the transformation prospects of engineering industry and the development of agricultural industry in construction industry, and examines the intellectual transformation of individual human capital in Chinese labor force. Article [28] assesses the financial performance of intelligent manufacturing enterprises by studying and analyzing the improvement of financial management model in intelligent manufacturing model and the optimization of cost involved. Article [29] studies the interaction and integration between the real world environment and the virtual space of the digital environment in order to realize the harmony between the virtual real space in the whole life cycle. Article [30] the engineering cost estimation model is studied, and the neural network comprehensive prediction model is established to improve the accuracy and application technology of the prediction model.

Article [31] studies the new computer technology to establish a Chinese teaching platform. The system is based on speech recognition technology to help foreigners learn spoken language, manuscript recognition technology and Chinese letters. Article [32] constructs an efficiency improvement model of English online teaching system. Article [33] proposes a hexahedral grid structure simplification method based on weighted sorting, which comprehensively sorts the elimination order of candidate base complexes in the grid with three sets of sorting items of width, deformation and price improvement. Article [34] explains the rules for the establishment of first-level courses, clarifies the ideas and priorities 
of architecture, and explores strategies for building university-level courses using knowledge of artificial intelligence and neural network algorithms in order to gain experience from them. Article [35] studies the application of better genetic algorithm to mine the relevant standards to improve the data acquisition technology. Article [36] evaluates the students IELTS writing ability, according to the established measurement and evaluation indicators. Article [37] discusses the new strategies of contemporary management accounting development based on the development of artificial intelligence technology and the combination of data mining and XBRL technology. Article [38] is based on learning a research on the innovation of enterprise financial management is carried out on machine and artificial intelligence technology. Article [39] summarizes the current situation of the development of digital teaching mode in colleges and universities in China; on the other hand, it analyzes the research results of computer teaching by professionals in the new era; finally, the knowledge of data mining is expounded. Article [40] uses a logarithmic spiral to simulate the path of the moth to the flame and invert the pending parameters in the mathematical model, and adds vertical and horizontal algorithms and chaos operators on this basis.

Article [41] discuss the spectral segments of plants, the spectral information of plant absorption and reflection in plants by means of artificial intelligence to provide data support for rape planting and management, and monitor the real-time status of its growth and development. Article [42] puts forward the design method of multi-sensor data fusion technology. It also introduces how to establish a new medical equipment system based on network registration, discovery and various management and fault-tolerant conditions. Article [43] discuss and study the development of graphic design by SVM theory. With the development of computer network technology, people promote the development of graphic design by constructing color management system. Article [44] designs an adaptive offloading decision algorithm ADCO and an online spring slide task scheduling algorithm SSLS based on a dynamic and heterogeneous English intelligent learning system. In Article [45], the sports video is taken as the analysis object, and the VIRE positioning algorithm uses linear interpolation to calculate the virtual label, when the error between the obtained RSSI value and the actual value is larger, this paper uses quadratic interpolation to improve it. Article [46] puts forward some viewpoints based on the current curriculum system in colleges and universities in order to improve the learning quality of computer courses. Article [47], On the basis of machine learning and the herd effect algorithm, an artificial intelligence-based English intelligent writing system is constructed. Article [48] tries out the practical application of machine learning feature extraction method in a certain perception system. Article [49] aims at word order detection in English classroom teaching, by proposing an analysis model based on block coding and improved genetic algorithm. Article [50] uses machine learning technology as the foundation and self-service data envelopment analysis to construct a comprehensive efficiency analysis model for the logistics industry.

Article [51] uses fuzzy preference relations and selects the main risk criteria to construct a risk evaluation index system. Article [52] proposes an identification system to assist or replace librarians in their daily book cataloguing work. A two-dimensional algorithm is used to segment the information element matrix to reduce sound. Article [53] constructs a computer English learning performance evaluation model based on machine learning. The computer English learning performance evaluation system is constructed on the basis of auditory characteristics and introduces a wavelet entropy feature based on the best tree wavelet packet decomposition, and it is applied to the establishment of an adaptive model. Article [54] builds an intelligent model of artistic design image modal analysis based on machine learning technology and image recognition technology, and proposes a feature extraction method based on wavelet transform and fuzzy logic and an art image classification method based on rotating quaternion wavelet transform. Article [55] takes some trees of the same growth year as the object of study. Using BP neural network to analyze the factors that will change the overall value of forest resources, a special BP neural network model belongs to forestry and economic development is established. Article [56] proposes a new video denoising algorithm, which uses the recently proposed grid flow motion model based on camera motion compensation to generate denoised video.

In conclusion, this special issue would not have been possible without the help of many people. As guest editors, we would like to take this opportunity to thank the authors for their contributions and the reviewers for their invaluable comments and timely responses. We also would like to thank the JIFS Editor-in-Chief and staff for their support during the preparation and production of this special issue. 